\title{
eTimeTrek: digital deep history of the Universe
}

\section{Kirsi Lehto', Anni Kauko", Pasi Nurmi², Natuschka Lee ${ }^{3}$, Elias Chatzitheodoridis $^{4}$ and Harry Lehto ${ }^{2}$}

${ }^{1}$ Molecular plant biology, Biochemistry department, and ${ }^{2}$ Department of physics and astronomy, University of Turku, Finland, ${ }^{3}$ Department of Ecology and Environmental Science, Umeå University, Sweden and ${ }^{4}$ School of Mining and Metallurgical Engineering, National Technical University of Athens, Greece

\section{Background}

The amount of knowledge is rapidly increasing in all fields of science, and our views and understanding of the world are constantly becoming deeper and more complicated. In the future the people will need sustainable education in all disciplines. The students must fully understand the fundamental principles of the world, in order to effectively participate in world forums, and make educated policies and good decisions. Such understanding will be required e.g. in areas related to new technologies and phenomena, such as those of space-sciences, nanoand DNA-technologies, artificial intelligence, automatization, climate and environmental issues, and even in societal issues such as overpopulation, aging, ever-increasing economic growth, and globalization. People also need to effectively deal with multiple sciences in order to achieve innovation, and to understand how different sectors function.

The flow of information is nowadays very high. This is a great challenge to the education system, which should provide this information in a comprehensive and understandable way, especially to the young students. The education should also be offered in an inspiring format, encouraging students to be actively involved in the scientific world and in our society. Our education system tends to follow the tradition of teaching each science topic in its own separate curriculum which leads to a fragmented view of the actual world, and sometimes the information may remain rather irrelevant to its surrounding world.

Meanwhile, the need of engaging learning and teaching methods has been widely recognised in the education field. The new learning theories state that the learning needs to be motivating and meaningful, and that sciences should be learned in an 
interdisciplinary setting, in the context of the phenomena that we actually observe in the real world. The learned information should be related to existing knowledge, and interactive discussions would allow the young learners to comprehend how all things relate to each other. Efficient learning happens in an interactive and immersive manner via different kinds of sensory observations (1).

The holistic view of our world, with all of its scientific phenomena could be presented in a comprehensive way by showing how they all fit into one long continuum of time. Many of them also occur in various cause-and-effect relationships and emerge in certain sequences, one on the top of the other: Our world has developed from its cosmic roots, based on the laws of the quantum physics, physics and chemistry, leading to ever increasing complexity of structures and interactions. Also, on the planetary level the chemical and physical processes determine the geological and climatological phenomena. In the early phases of this planet these led to the emergence of life via inorganic/organic prebiotic chemistry. Since then, the biosphere has flourished with ever increasing complexity of forms and functions, and it has also largely affected the conditions on this planet. Still, the existence of the biosphere continues only in very close balance and with interactions with the environmental conditions. It is totally dependent on the ever-continuing inflow of energy, on the large global cycles of elements that are needed for the synthesis of biomass, and on the stable flow of the produced biomass through the food chains and degradation processes.

The fundamental natural processes and phenomena occur in huge cosmic and universal scales 1 (e.g. the principles of physics and chemistry, and all natural laws), while many function also in the planetary scale (e.g. the factors that determine our climate, or the rules of evolution). Still, many of their essential functions and reactions happen in quantum-, atomic-, or nanoscales. These hugely different scales and dimensions are difficult to comprehend. So are also the hugely varying time scales where our world has developed. The time scales vary from fractions of the second (as in the Big Bang) to the life times of stars (several billions of years), to the time of continental movements and collisions (several millions of years), to the time of cell division and regenerations of different species (from hour to some hundreds of years). Comprehension of the spatial and time dimensions would be useful for understanding the present state of the world, and furthermore, to help the students relate all their learning to this holistic view of the world. 
We have constructed a holistic view and a framework of the world's time dimensions by developing "Timetrek", i.e., a $13.7 \mathrm{~km}$ long walkable timeline that portrays the history of the universe, the Earth and life (2). This walkable timeline enables a "walkable" experience that focuses on the time scales of the natural processes. Every meter on the trail corresponds to a million years, and every kilometre to a billion years of the history of the world. The Timetrek trail runs from the Tuorla observatory to the Turku University main campus, ending there next to a statue of Eric Copper called "Echo of the Big Bang", a statue with loudspeakers that amplify the hum of the cosmic background radiation. The main events in the natural history have been marked in information plates along the route, and additional illustrated and narrated information is provided for each time point on the webpage timetrek.aikavaellus.fi/timeline. These pages can be easily accessed also via QR codes at each time point.

The Timetrek tells the deep story of how our world has developed, and it allows us to imagine the different rates of on-going cosmic and terrestrial processes. For instance, large mountain chains are being lifted up and worn down, as shown within some tens of meters along the trail. The continents are drifting on the sea bottoms, and slowly, but always, they collide to each other, while the sea floor subducts gradually into the deep layers of the mantle. The tectonics, driven by these processes, cycle carbon between its reservoirs in the atmosphere, the ocean sediments and the Earth mantle. The continental positions affect the albedo and thus the temperature of the planet. All these affect the atmospheric composition and the climate, which again interferes with the carbon cycle in slow feed-back loops. Additionally, the biosphere with its biomass production and consumption, and nowadays through the human activities, strongly affect these processes.

Timetrek informs also about the other essential natural conditions and processes which have enabled all the major developments of the biosphere. Within its last centimetres it also brings up the human history. This period is given a special focus and is being justified as a point of interest for humans, mainly because our species is now causing major changes in the conditions of the whole planet. Our place on the timeline indicates that the development of human civilization started only 10000 years ago, or only $1 \mathrm{~cm}$ from the time point that represents the presence. The beginning of the organized agriculture and communal living was related to severe droughts at that time, but after that has continued in very favourable climate conditions. 
Throughout its history the conditions on our planet have spanned from hot-house conditions with ocean levels about 100-200 meters higher than today's level, to different levels of glaciations with significantly lower ocean levels compared to today. Both these situations appeared semi-stable during the time they prevailed, but when the atmospheric composition or the continental positions will start to change, then this balance can change abruptly. The large changes in planetary conditions are associated with the periodic extinction of certain biological species, even through mass extinctions. Time after time, these large changes have provided new opportunities for new species to emerge, thus driving the radiative evolution and the diversification of the biosphere.

These views should be considered as provoking, as they illustrate how unified our mankind is here, by existing on one small globe and breathing the same atmosphere, while they depend on a single fragile climate that is favourable for biological production.

\section{Timetrek as a teaching material for schools}

The walkable Timetrek is a trail where the students can experience the continuum of the natural processes, and feel the dimensions of time as physical exercise in their feet as they walk. The whole walk is possible to make in a single full-day outing of a school, but the walking can also be made in shorter stretches, e.g., the 4,5 km distance for the whole history of the Earth, or the $2 \mathrm{~km}$ distance for the existence of multicellular life, both suitable for thematic classes of specific topics. The walking format provides the outing and physical exercise to be connected to learning, but it also gives the opportunity for discussing the topics while the students walk.

Scalability of the timeline: The current scale of the Timetrek now covers 1 million years in each meter. However, this scale can be easily changed, e.g. by condensing the whole distance by a factor of 10 , to fit the whole trail into the school yard. The scale could be also expanded by a factor of 10, i.e. expand the last 100 meters to a distance of $1 \mathrm{~km}$, or by 100 fold to expand the last meter into 100 meters, to build up of reasonable resolution for the evolution of our current species, or humans, respectively. These could be tasks for the students and classes to build up by themselves, as assisted by the information available from the Timetrek project. The students could also include in the trails any new events and time points that they find important - or build new timelines for different themes e.g., related to the human history. 
Transportability: Since all the learning material is provided as on-line material the Timetrek trail is not bound to a particular location, but can be easily transferred to anywhere else. Some teachers have indicated that it would be most useful and functional for teaching purposes if it would be constructed by the students themselves, into their own school surroundings where they can easily comprehend the distances. Illustrative is to understand that if the Universe was formed in the village next to their own which for example is about 14 kilometres from their school, then, the Solar system and the Earth have been formed at a distance of 4,5 $\mathrm{km}$, and complex life appeared at 500 meter away. The dinosaurs then died at a distance of only 66 meters, while the last ice age ended only one centimetre before the school's position (or time point). These time points could be designated in a suitable walking trail in the vicinity of any school, and marked with the QR codes of the Timetrek project in order to get access to the original information pages.

Walk your own Timetrek routes: Still a more flexible set-up for walking on the Timetrek route will be available via a new geo-location mobile application. This app keeps track of the distance one has walked, defined on "his/her own" Timetrek, and gives a signal whenever you have reached the next time point. The application plays the recorded narrations for each time point as you walk to the right distance to reach them; the application can even keep record of your performance, allow you continue "your own Timetrek" the next day, and collect "points" of the distances you walk.

Although the long Timetrek walk is a good way to demonstrate the Deep History issues with physical exercise, there is also a need to reveal this holistic concept in a more illustrated, descriptive and interactive way. We have already started to produce a digital, gamified and illustrated pilot material to be used as the gamified Timetrek story:

https:/drive.google.com/drive/folders/0B57nIuVXkcyeM1VnRWFUbmt4ZEU. In this pilot material we have so far produced only the episode portraying the planetary history, but here we have aimed to charm the students with the beauty of science. We have also used modelling to promote understanding of the planetary processes.

We think that these new educational methods need to be addressed with a bold, creative, interdisciplinary and holistic approach, by combining science teaching with arts, games, testing and experimentation, physical exercise and future technologies. The learning platform should also be modular and interactive in such a way that scientists can easily provide new pieces of data, teachers can modify the material at will, and students can be engaged in seeking up information and giving their own input. 


\section{References:}

1. Teaching guide for GSI. Available at http://gsi.berkeley.edu/gsi-guidecontents/learning-theory-research/learning-overview/

2. Lehto, K., Lehto, H. et al. 2013. Time Trek: a $13.7 \mathrm{~km}$ long nature trail leading through the history of the Universe and the Earth. Int. J. of Astrobiology 12:1-7. 